Accepted for Publication in ApJ: July 11, 2013

Preprint typeset using $\mathrm{L}_{\mathrm{A}} \mathrm{EX}$ style emulateapj v. 5/2/11

\title{
RESOLVING THE MOTH AT MILLIMETER WAVELENGTHS
}

\author{
Angelo Ricarte ${ }^{1}$, Noel Moldvai ${ }^{1}$, A. Meredith Hughes ${ }^{2}$, Gaspard Duchêne $^{1,3}$, Jonathan P. Williams ${ }^{4}$, Sean M. \\ ANDREWS $^{5}$, DAVID J. WILNER ${ }^{5}$ \\ Accepted for Publication in ApJ: July 11, 2013
}

\begin{abstract}
HD 61005, also known as "The Moth," is one of only a handful of debris disks that exhibit sweptback "wings" thought to be caused by interaction with the ambient interstellar medium (ISM). We present $1.3 \mathrm{~mm}$ Submillimeter Array (SMA) observations of the debris disk around HD 61005 at a spatial resolution of $1^{\prime \prime} .9$ that resolve the emission from large grains for the first time. The disk exhibits a double-peaked morphology at millimeter wavelengths, consistent with an optically thin ring viewed close to edge-on. To investigate the disk structure and the properties of the dust grains we simultaneously model the spatially resolved $1.3 \mathrm{~mm}$ visibilities and the unresolved spectral energy distribution. The temperatures indicated by the SED are consistent with expected temperatures for grains close to the blowout size located at radii commensurate with the millimeter and scattered light data. We also perform a visibility-domain analysis of the spatial distribution of millimeter-wavelength flux, incorporating constraints on the disk geometry from scattered light imaging, and find suggestive evidence of wavelength-dependent structure. The millimeter-wavelength emission apparently originates predominantly from the thin ring component rather than tracing the "wings" observed in scattered light. The implied segregation of large dust grains in the ring is consistent with an ISM-driven origin for the scattered light wings.

Subject headings: circumstellar matter — stars: individual (HD 61005) — Submillimeter: planetary systems
\end{abstract}

\section{INTRODUCTION}

Debris disks around main sequence stars provide an indicator that planet formation has proceeded at least to the scale of planetesimals. All three directly imaged planetary systems to date (Fomalhaut, HR 8799 , and $\beta$ Pictoris; Kalas et al. 2008; Marois et al. 2008; Lagrange et al. 2010) also host debris disks, and in two cases disk structure (eccentricity and warping) led to the prediction of at least one perturbing body in the system (e.g. Heap et al. 2000; Burrows et al. 1995). Investigations of debris disk structure are therefore an important facet in our understanding of extrasolar planetary systems. Millimeter-wavelength imaging plays an important role, since it provides access to a population of large dust grains that responds primarily to the gravitational dynamics of the system, rather than the radiation forces that sculpt the small grains that dominate optical and infrared observations (Wyatt 2006). Multiwavelength observations of nearby systems have begun to reveal wavelength-dependent structure including extended haloes of small grains (e.g. Su et al. 2009), often apparently generated by a much more radially confined ring of large parent bodies (Wilner et al. 2011, 2012).

HD 61005 (also known as "The Moth") is part of an intruiguing sample of debris disks with swept-back

\footnotetext{
${ }^{1}$ University of California Berkeley, Department of Astronomy, 601 Campbell Hall, Berkeley, CA 94720

${ }^{2}$ Wesleyan University Department of Astronomy, Van Vleck Observatory, 96 Foss Hill Drive, Middletown, CT 06459

${ }^{3}$ UJF-Grenoble $1 /$ CNRS-INSU, Institut de Planétologie et dAstrophysique de Grenoble (IPAG) UMR 5274, BP 53, 38041 Grenoble Cedex 9, France

${ }^{4}$ Institute for Astronomy, University of Hawaii, etc.

${ }^{5}$ Harvard-Smithsonian Center for Astrophysics, 60 Garden Street, Cambridge, MA 02138
}

features believed to result from interactions with the ambient interstellar medium (the others are HD 32297 and HD 15115; Kalas 2005; Debes et al. 2008, 2009; Rodigas et al. 2012). The disk has been imaged in scattered light both from the ground and in space, revealing a thin ring with a possible position offset from the central star and a symmetric pair of streamers originating from the ring ansae (Hines et al. 2007; Maness et al. 2009; Buenzli et al. 2010). Several mechanisms have been proposed to explain the unusual morphology of these sweptback debris disks, including two distinct ISM-driven mechanisms that result in similar morphology despite the orthogonal directions they assume for stellar motion relative to the ambient medium (Maness et al. 2009; Debes et al. 2009). By contrast, Marzari \& Thébault (2011) determine that the effects of the ISM are extremely sensitive to geometrical optical depth (which is directly proportional to the collision rate) and grain size, so that only the morphology of disks with low optical depths imaged at short wavelengths should be noticeably affected by interactions with the ISM. If the morphology persists for high optical depths or large grain sizes, then alternative mechanisms such as dynamical sculpting by an embedded planet should be considered. It is therefore desirable to image debris disks with swept-back structures at millimeter wavelengths, in order to determine the morphology of large grains in the system and thereby investigate the physical origins of the observed structure.

HD 61005 presents an attractive target for millimeterwavelength imaging. Located in the local bubble (Franco 1990) at a distance of $34.5 \mathrm{pc}$ (Perrvman et al. 1997) and exhibiting a ring radius of $61 \mathrm{AU}$ (Hines et al. 2007; Buenzli et al. 2010), it spans spatial scales that are amenable to being resolved with an interferometer. It 

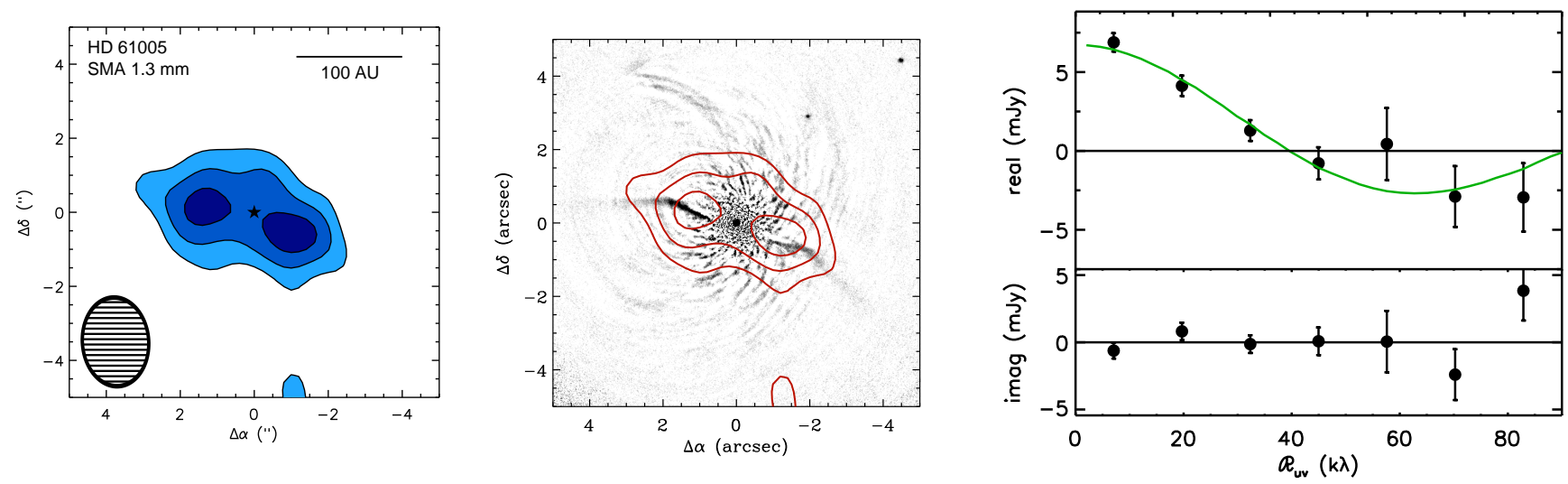

Fig. 1. - Left: SMA map of the $1.3 \mathrm{~mm}$ emission from the debris disk around HD 61005. The image has been reconstructed using all data with both array configurations. The axes have been set such that the $(0,0)$ position corresponds to the expected location of the star at the epoch of observation (with each data set individually corrected for proper motion), and its position is marked by a star symbol. Contours are drawn at $[3,5,7] \times 0.34 \mathrm{mJy}$ (the rms noise). An ellipse indicating the size of the synthesized beam $\left(2^{\prime \prime} 5 \times 1^{\prime \prime} \cdot 9\right.$ at a position angle of $4^{\circ}$ ) is displayed in the bottom left corner. The emission is resolved into two distinct peaks, consistent with the expected morphology for an optically thin debris disk viewed close to edge-on. Center: $1.3 \mathrm{~mm}$ contours overlaid onto H-band image Buenzli et al. (2010). These images were aligned by eye due to uncertainties in the pointing coordinates of the optical image. The relatively low resolution of the SMA data collects flux from along the disk major axis and causes the peaks to appear closer together than the ansae of the scattered light ring. Right: $1.3 \mathrm{~mm}$ visibilities plotted as a function of the baseline length, deprojected to account for the $84.3^{\circ}$ inclination angle at which the source is viewed (see, e.g., Hughes et al. 2008, for a mathematical description of the abscissa). The green line shows the visibilities for the best-fit model.

has the largest $24 \mu \mathrm{m}$ excess of all main sequence stars observed in the FEPS (Formation and Evolution of Planetary Systems) legacy survey on the Spitzer Space Telescope (Mever et al. 2008), and a bright $350 \mu \mathrm{m}$ flux of $95 \pm 12 \mathrm{mJy}$ (Roccatagliata et al. 2009), which predicts a substantial millimeter-wavelength flux for imaging. As a Solar analogue (spectral type G 3/5 V; Hillenbrand et al. 2008) it is of particular interest for understanding the history of our own solar system at younger ages. Stellar age estimates have varied around $100 \mathrm{Myr}$, but recent work suggests that it is likely a member of the Argus association (Desidera et al. 2011). Membership in the Argus association would lower its age to $40 \mathrm{Myr}$, which is more consistent with its large $24 \mu \mathrm{m}$ excess.

Here we use interferometric millimeter-wavelength imaging with the Submillimeter Array (SMA) to investigate the wavelength-dependent structure of the disk around HD 61005. In Section 2, we present SMA observations at a wavelength of $1.3 \mathrm{~mm}$ that resolve the structure of the large grain populations for the first time. We analyze the spectral energy distribution (SED) and interferometric visibilities simultaneously to study the spatial distribution and thermal properties of the grains in Section 4. In Section [5 we discuss the implications of our observations in the context of proposed mechanisms for creating swept-back structures. In Section 6 we summarize the main results of our investigation and emphasize the value of future observations for providing insight into the underlying physical processes shaping the system.

\section{OBSERVATIONS}

HD 61005 was observed with the SMA for four nights between 2008 December and 2012 January. The basic observational parameters are listed in Table 1 . The first three nights of observation were carried out in the SMA's compact configuration, while the final night was conducted in the extended configuration (see table for baseline lengths). Due to the southern declination of the source, the spatial resolution was generally finer in the east-west direction than the north-south direction. The weather was generally very good, with low $225 \mathrm{GHz}$ opacity, indicating high atmospheric transparency to millimeter-wavelength radiation, and stable atmospheric phase. The quasar J0747-331, located only $2.6^{\circ}$ away from HD 61005, was used as the gain calibrator for all four tracks to correct for atmospheric and instrumental variations in amplitude and phase; the derived flux for the quasar is listed in the final column of Table 1. Observations of the gain calibrator and source were interleaved with the quasar J0826-225, which was used to test the efficacy of the phase transfer. Bandpass calibration was carried out using a bright quasar $(3 \mathrm{c} 84,3 \mathrm{c} 273$, or 3c454.3). The absolute flux scale was set using solar system objects (specified for each track in Table 1); we assume a standard (but conservative) $20 \%$ systematic flux uncertainty due to uncertainties in the flux models for these objects. The correlator was configured to maximize continuum sensitivity by utilizing the largest available bandwidth. The total bandwidth in the 2008 track is $2 \mathrm{GHz}$ per sideband, with a sideband separation of $\pm 5 \mathrm{GHz}$ from the local oscillator (LO) frequency listed in the table. The bandwidth was upgraded to $4 \mathrm{GHz}$ per sideband for the three subsequent tracks. Routine calibration tasks were carried out using the MIR software package 6 , and imaging and deconvolution were accomplished with MIRIAD.

\section{RESULTS}

We detect the disk around HD 61005 independently on all four nights. The combined map and visibilities are displayed in Figure 1. The disk is strongly detected, with a peak signal-to-noise ratio of $\sim 8$ in two separate beams. An appropriate shift to the visibilities has been applied to each data set to account for the proper motion

${ }^{6}$ See http://cfa-www.harvard.edu/ cqi/mircook.html 
TABLE 1

SMA Observational Parameters

\begin{tabular}{|c|c|c|c|c|c|c|c|c|c|}
\hline Date & Antennas & $\begin{array}{c}\text { Baselines } \\
(\mathrm{m})\end{array}$ & $\tau_{225 \mathrm{GHz}}$ & $\begin{array}{l}\text { LO Freq } \\
(\mathrm{GHz})\end{array}$ & $\begin{array}{c}\text { RMS noise } \\
\left(\mathrm{mJy}^{a} \text { beam }^{-1}\right)\end{array}$ & $\begin{array}{c}\text { Synthesized Beam } \\
(\operatorname{arcsec})\end{array}$ & Flux Cal & Gain Cal & $\begin{array}{c}\text { Derived Flux } \\
(\mathrm{Jy})\end{array}$ \\
\hline 2008 Dec 16 & 7 & $16-68$ & $0.10-0.15$ & 225.497 & 1.1 & $6.5 \times 2.8$ & Uranus & J0747-331 & 0.90 \\
\hline 2009 Dec 29 & 8 & $16-77$ & 0.06 & 225.169 & 0.6 & $5.7 \times 2.9$ & Uranus/Titan & J0747-331 & 0.80 \\
\hline 2010 Apr 13 & 8 & $16-69$ & 0.04 & 225.169 & 0.8 & $6.2 \times 3.0$ & Titan & J0747-331 & 1.02 \\
\hline 2012 Jan 29 & 7 & $50-226$ & $0.05-0.09$ & 225.497 & 0.7 & $2.1 \times 1.9$ & Uranus & J0747-331 & 0.95 \\
\hline
\end{tabular}

REFERENCES. - a Naturally weighted image

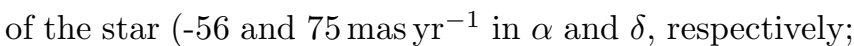
van Leeuwen 2007). The centroid of the millimeterwavelength emission is consistent to within the uncertainties with the expected J2000 position of the star $(\alpha$ $\left.=7^{h} 35^{m} 47.462^{s}, \delta=-32^{\circ} 12^{\prime} 14.043^{\prime \prime}\right)$.

The IR excess from the literature suggests that the disk is optically thin, and the double-peaked structure we observe is consistent with the expected morphology for a highly-inclined disk with a central cavity. While the structure is clearly not well described by a Gaussian, we estimate some basic structural parameters by fitting an elliptical Gaussian to the visibilities using the MIRIAD task uvfit. The fit yields a major axis of $4^{\prime \prime} 4 \pm 0^{\prime \prime} .6(150 \pm 20 \mathrm{AU})$ and a minor axis of $0 . .03 \pm 0^{\prime \prime} 6$ $(0.1 \pm 20 \mathrm{AU})$, implying that the disk is spatially resolved along the major axis but not along the minor axis. The position angle is $71^{\circ} \pm 5^{\circ}$, consistent with the value obtained from scattered light imaging, $70.3^{\circ}$ with a $\sim 1^{\circ}$ uncertainty (Buenzli et al. 2010). The integrated flux estimated from the elliptical Gaussian fit is $7.9 \pm 0.7 \mathrm{mJy}$, although a far more reliable value will be obtained in the MCMC analysis in Section 4.2. Buenzli et al. (2010) also report that the center of the ring is offset from the star along the major axis by $2.75 \pm 0.85$ AU toward the SW peak. We measure the offset of the center of the Gaussian compared to the star's position, corrected for proper motion. The centroid of the Gaussian is consistent with the star position to within $\pm 7 \mathrm{AU}$; the uncertainties are too large to confirm the offset observed in scattered light by Buenzli et al. (2010).

\section{ANALYSIS}

To determine both the geometric properties of the disk and thermal properties of the constituent dust we simultaneously model the Spectral Energy Distribution (SED) and the resolved $1.3 \mathrm{~mm}$ visibilities (Section 4.1). The best fit models are obtained from Markov Chain Monte Carlo fitting (Section 4.2). We begin by fitting a geometrically thin ring, motivated by the observed scattered light morphology. To place the results of MCMC fitting in context, we then investigate the effects of the assumed dust properties (Section 4.2.1) and ring width (Section 4.2.2). We also conduct a visibility-domain comparison of the millimeter-wavelength emission with the known scattered light morphology to investigate the relative spatial distribution of small and large grains (Section 4.3).

\subsection{Disk Model}

As shown in Figure 2, we model the SED with three components: (1) a Kurucz-Lejeune model photosphere with surface gravity $\log g=4.5$, effective temperature $T_{\text {eff }}=5500 \mathrm{~K}$, and solar metallicity $\mathrm{Z}=0.01$ (Desidera et al. 2011), (2) a cold, spatially-resolved outer debris disk, and (3) a warm inner dust belt modeled as a single-temperature blackbody. The addition of the belt is necessary to increase the flux around 20 microns, where neither the star nor the disk contribute enough flux to account for observations. The short-wavelength excess that we attribute to the presence of an inner belt could equally arise from hot emission from a population of grains smaller than the characteristic grain size in our model; however, the data at this point are insufficient to distinguish between the two scenarios. Accordingly, the properties of the warm belt are not well constrained, since it only produces a substantial contribution to the total flux over very small portion of the observed range of wavelengths, so for simplicity we allow only the mass of the belt to vary, and parameterize it as a narrow ring of $100 \mathrm{~K}$ dust (we also demonstrate below that our results are not sensitive to the assumed temperature). The cold disk, which we model as a spatially extended component, contributes essentially all of the flux at $1.3 \mathrm{~mm}$; in our best fit, the warm dust belt contributes only $0.36 \mathrm{mJy}$ to the total flux at this wavelength, a factor of 20 less than that of the extended component. This modeling procedure is similar to that presented in Hughes et al. (2011), with the primary difference being the use of astrosilicate opacities to determine grain temperatures. We briefly describe the salient features of the model below.

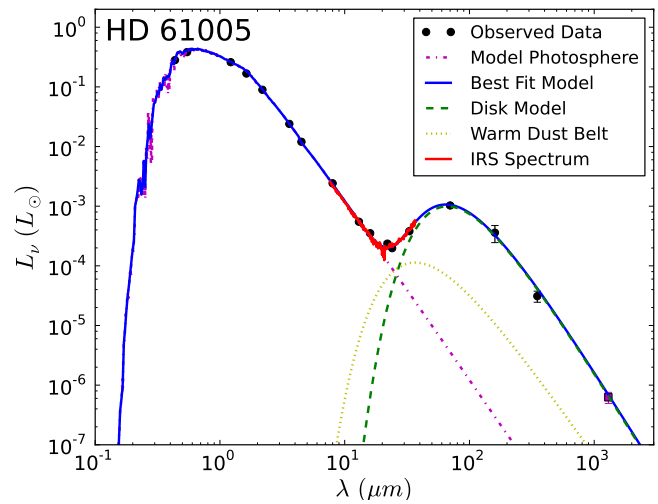

FIG. 2.- SED of the HD 61005 system. The total model SED is the sum of three components: a Kurucz-Lejeune model photosphere, the debris disk, and a warm $100 \mathrm{~K}$ dust belt. The flux we measure at $1.3 \mathrm{~mm}$, which is calculated from our best fit model and is not included in the calculation of the SED $\chi^{2}$ value, is displayed with a magenta square. The error bars for this point include an estimated $20 \%$ systematic uncertainty. The units are defined as $L_{\nu}=4 \pi d^{2} \nu F_{\nu}$ in units of $L_{\odot}$. Our model coincides well with the Spitzer IRS spectrum, despite the fact that these data were not used in the fitting process and are displayed for comparison only.

Each disk model is determined by 6 free parameters: the inner radius of the disk $\left(R_{i n}\right)$, the characteristic grain 
size $(a)$, the disk mass $\left(M_{D}\right)$, the grain emissivity parameter $(\beta)$, the belt mass $\left(M_{B}\right)$, and the width of the disk $(w)$. In our initial fitting efforts, we fix the width of the disk $w$ to a small, constant fraction of $5 \%$ of $R_{\text {in }}$ to match the scattered light morphology (although this assumption is relaxed in Section 4.2.2 below). $R_{i n}$ is mostly constrained by the visibilities, but it also has an effect on the equilibrium temperature of the dust grains, which directly affects the peak flux and wavelength of the SED. The grain size, $a$, determines the temperature of the grains. In reality the disk most likely has a broad distribution of grain sizes, however assumption of a single characteristic grain size is sufficient to reproduce the observed SED while minimizing computational requirements. $M_{D}$ is essentially a luminosity scaling factor for the disk SED, just as $M_{B}$ scales the flux of the belt. Finally, $\beta$ determines the slope of the Rayleigh-Jeans tail. In accordance with Williams et al. (2004), we assume a dust grain emission efficiency $Q_{\lambda}=1-\exp \left[-\left(\lambda / \lambda_{0}\right)^{-\beta}\right]$, where $\lambda_{0}=2 \pi a$ is the critical wavelength. This function has the desired asymptotic properties that $Q_{\lambda} \approx$ $\left(\lambda / \lambda_{0}\right)^{-\beta}$ when $\lambda>>\lambda_{0}$, and $Q_{\lambda} \approx 1$ when $\lambda<<\lambda_{0}$. This parametrization of $Q$ does not factor into our calculation of the grains' temperature, however. It is required to ensure smooth long-wavelength emission in the SED by mimicking the effect of a grain size distribution, since grains at the characteristic grain size are extremely inefficient emitters at $\lambda>>a$.

In order to determine the temperature of a grain of a given size and distance from the star, we assume that the dust composition is compact astrosilicates (Draine 2003) and obtain the grains' opacity, $\kappa_{\text {tot }}(a, \lambda)$, and albedo, $\omega(a, \lambda)$, using Mie theory (see, e.g. Bohren \& Huffman 1983) as implemented in the radiative transfer code MCFOST (Pinte et al. 2006, 2009). We then derive the appropriate grain temperature from energy balance. As written in Tielens (2005), the energy emitted per unit time from the grain can be expressed:

$$
\Gamma_{\text {out }}=4 \pi \cdot \pi a^{2} \cdot \int_{0}^{\infty} Q(a, \lambda) B(T, \lambda) d \lambda
$$

where $Q(a, \lambda)$ represents the fraction of emission at wavelength $\lambda$, and $B(T, \lambda)$ is the grains' Planck function. Similarly, the power absorbed from the star can be written:

$$
\Gamma_{i n}=\pi a^{2} \cdot \int_{0}^{\infty} Q(a, \lambda) F_{\lambda}(r, \lambda) d \lambda
$$

where $F_{\lambda}$ is determined from our Kurucz-Lejeune model of the stellar photosphere, $a$ is the grain size, and $r$ is the distance from the star to the grain. Rather than using the simplistic parametrization of $Q$ mentioned above to determine the flux density of the Rayleigh-Jeans tail, we obtain $Q(a, \lambda)$ from $\kappa_{\text {tot }}(a, \lambda)$ and $\omega(a, \lambda)$. Assuming spherical grains, the absorption efficiency is:

$$
Q(a, \lambda)=\frac{4}{3} \kappa_{t o t}(a, \lambda) \rho a(1-\omega(a, \lambda))
$$

where $\rho$ is the mass density of a single grain.Setting $\Gamma_{i n}=$ $\Gamma_{\text {out }}$, we arrive at:

$$
\frac{1}{4 \pi} \int_{0}^{\infty} Q(a, \lambda) F_{\lambda}(r, \lambda) d \lambda=\int_{0}^{\infty} Q(a, \lambda) B(T, \lambda) d \lambda
$$

By numerically evaluating each integral in Equation 4, we obtain the temperature of a given grain. In order to do so, we generate a lookup table of these integrals. The integral on the left is tabulated as a function of $a$ and calculated at a radius of $50 \mathrm{AU}$; to determine the values of this integral at different radii, we scale these values by $1 / r^{2}$. The second integral is tabulated as a function of both $a$ and T. $a$ is sampled from 0.1 to 3000 microns with about 3 sizes per decade (in log space), and $T$ is sampled from 0 to $1000 \mathrm{~K}$ with a step size of $1 \mathrm{~K}$. The associated wavelengths in our lists of $\kappa_{\text {tot }}(a, \lambda)$ and $\omega(a, \lambda)$ span from $10^{-5}$ to $3 \times 10^{-1} \mathrm{~cm}$, sampled in logarithmic intervals of 0.05 .

The surface number density of grains, $N(r)$, is related to the surface mass density as $\Sigma(r)=N(r) m_{g}$, where $m_{g}$ is the mass of a grain, which we assume is spherical. We assume a density of $2.7 \mathrm{~g} \mathrm{~cm}^{-3}$, which is a compromise between typical bulk densities measured for cometary and interplanetary dust particles and terrestrial materials typically assumed to comprise astronomical graphite or silicate grains (see, e.g., Draine \& Lee 1984; Brownlee et al. 2006; Blum \& Wurm 2008). We then parameterize $\Sigma(r)=\Sigma_{100}\left(\frac{r}{100 \mathrm{AU}}\right)^{-p}$, where $p$ is the surface density power law, which we fix at a value of 1 (this value is consistent with both the radial falloff of surface brightness in the region of the AU Mic birth ring postulated as the birth ring in Strubbe \& Chiang (2006), as well as a typical value for bright protoplanetary disks measured by Andrews et al. (2009)). There is a well-known degeneracy between $p$ and the outer radius, and our data are not of sufficiently high quality to distinguish between these variables; see, e.g., discussion in Mundy et al. (1996). We integrate flux contributions between the inner and outer radius, yielding:

$$
F_{\lambda}=\frac{\pi a^{2} Q(\lambda)}{d^{2}} \int_{R_{\text {in }}}^{R_{\text {out }}} 2 \pi r B_{\lambda}\left(T_{r}\right) N(r) d r
$$

At the observed wavelength of $1.3 \mathrm{~mm}$, we use the equations above to generate a high-resolution synthetic image of the disk. As is evident in Figure 2, the star and the $100 \mathrm{~K}$ dust belt do not contribute significant flux at the wavelength of the SMA observations, and are hence absent from the model image. To generate this image, we assume an inclination angle of $84.3^{\circ}$ and a position angle of $70.3^{\circ}$ derived from the higher-resolution scattered light images (Buenzli et al. 2010), and project the flux onto the sky plane. We then utilize the MIRIAD task uvmodel in order to sample the model image at the same spatial frequencies as the SMA data and compare the data and model in the visibility domain.

\section{2. $M C M C$ Fitting}

In order to compare the model with the SED and visibilities, we compute a $\chi^{2}$ value for each and sum the two. As discussed in Andrews et al. (2009), these two values of $\chi^{2}$ are comparably sensitive to changes in the disk parameters (the large numbers of visibilities are balanced by the low fractional uncertainty on the SED points), causing neither to dominate the total $\chi^{2}$ and thus the final fit. We omit the SMA $1300 \mu \mathrm{m}$ flux from the SED $\chi^{2}$ calculation since it is implicitly included in the visibility $\chi^{2}$. In order to locate the best fit and determine constraints on each parameter, we utilize the Markov 
Chain Monte Carlo (MCMC) fitting technique described in Goodman \& Weare (2010), an affine-invariant ensemble sampler which performs well when parameters are correlated. The SED includes the observed fluxes listed in Table 2. Only the points with wavelengths beyond 10 $\mu \mathrm{m}$ are affected by the parameters of the model disk, and therefore we include only these values in the computation of $\chi^{2}$.

TABLE 2

Observed Flux Points for HD 61005

\begin{tabular}{ccc}
\hline$\lambda(\mu \mathrm{m})$ & Flux (Jy) & Source \\
\hline 0.436 & $1.04 \pm 0.02$ & Tycho-2 (Høg et al. 2000) \\
0.545 & $1.82 \pm 0.02$ & Tycho-2 (Høg et al. 2000) \\
1.220 & $2.77 \pm 0.07$ & 2MASS (Cutri et al. 2003) \\
1.630 & $2.39 \pm 0.10$ & 2MASS (Cutri et al. 2003) \\
2.190 & $1.71 \pm 0.04$ & 2MASS (Cutri et al. 2003) \\
3.6 & $0.75 \pm 0.02$ & FEPS (Hillenbrand et al. 2008) \\
4.5 & $0.47 \pm 0.01$ & FEPS (Hillenbrand et al. 2008) \\
8 & $0.17 \pm 0.004$ & FEPS (Hillenbrand et al. 2008) \\
13 & $0.062 \pm 0.004$ & FEPS (Hillenbrand et al. 2008) \\
16 & $0.049 \pm 0.003$ & Spitzer IRS Archive \\
22 & $0.045 \pm 0.004$ & Spitzer IRS Archive \\
24 & $0.041 \pm 0.002$ & FEPS (Hillenbrand et al. 2008) \\
33 & $0.11 \pm 0.007$ & FEPS (Hillenbrand et al. 2008) \\
70 & $0.63 \pm 0.05$ & FEPS (Hillenbrand et al. 2008) \\
160 & $0.50 \pm 0.16$ & FEPS (Hillenbrand et al. 2008) \\
350 & $0.095 \pm .012$ & CSO (Roccatagliata et al. 2009) \\
\hline
\end{tabular}

After experimentation with the initial values of the ensemble, we find that the ensemble consistently converges to the same region in the parameter space independent of the initial parameter values. For all subsequent runs, we fix the initial values of the chain to the values of a reasonably good fit: $R_{i n}=68 \mathrm{AU}, \log (a[\mu m])=0.5$, $\log \left(M_{D}\left[M_{\oplus}\right]\right)=-2.7, \beta=0.5, \log \left(M_{B}\left[M_{\oplus}\right]\right)=-6.0$. Trial states for $R_{i n}$ and $\beta$ are generated in linear space, while states for $a, M_{D}$, and $M_{B}$ are generated in logarithmic space. The widths of the Gaussians determining trial states for each parameter are set to $2 \mathrm{AU}$ for $R_{\text {in }}, 0.05$ for $\log (a), 0.1$ for $\log \left(M_{D}\right), 0.01$ for $\beta$, and 0.05 for $\log \left(M_{B}\right)$, which we found were the approximate uncertainties in these parameters. We run 100 "walkers" through 800 trials each, and after rejecting the "burn-in" phase (the region in which the average $\chi^{2}$ decreases with time before settling) which constitutes the first $\sim 200$ trials, we then determine the best-fit model and generate probability distributions for each of the parameters.

Best fit parameters for the five free parameters are listed in the left column of Table 3 . Figure 3 displays probability density functions generated by the models of the chain, as well as the locations of the best fit parameters, defined as the mode of the probability distributions. Figure 4 displays the best fit model image, as well as its residuals. For this best fit, we obtain a total flux of $7.2 \pm 0.3 \mathrm{mJy}$ (with an additional estimated $20 \%$ systematic uncertainty). For this value, and the values in Table 3. the uncertainty is determined from the posterior PDF of the ensemble as the width in parameter space which encloses $68.2 \%(1 \sigma)$ of the models, which we found to be symmetric about the best fit. For each of these models, the reduced $\chi^{2}$ is equal to 1.85 .

There are known degeneracies in this fitting process.
TABLE 3

The Best FIT PARAMETERS FOR HD 61005.

\begin{tabular}{cccc}
\hline Parameter & Best-Fit Model & For $T_{B}=80 \mathrm{~K}$ & For $w=65 \mathrm{AU}$ \\
\hline$R_{\text {in }}(\mathrm{AU})$ & $67 \pm 2$ & $67 \pm 2$ & $71^{a} \pm 3$ \\
$\log (a[\mu \mathrm{m}])$ & $0.38 \pm 0.07$ & $0.44 \pm 0.09$ & $0.33 \pm 0.09$ \\
$\log \left(M_{D}\left[M_{\oplus}\right]\right)$ & $-2.92 \pm 0.13$ & $-2.92 \pm 0.17$ & $-2.91 \pm 0.16$ \\
$\beta$ & $0.43 \pm 0.05$ & $0.40 \pm 0.06$ & $0.41 \pm 0.06$ \\
$\log \left(M_{B}\left[M_{\oplus}\right]\right)$ & $-5.93 \pm 0.06$ & $-5.23 \pm 0.06$ & $-5.97 \pm 0.08$ \\
Total $\chi^{2}$ & 456103.228 & 456107.180 & 456107.413 \\
\hline
\end{tabular}

REFERENCES. - a This is the radius which encloses half of the total flux.

Since we parameterized the disk with a single characteristic grain size, for a given value of $M_{D}$, increasing $a$ decreases the number of blackbody emitters and decreases their temperature, which therefore decreases the total flux. Hence, $M_{D}$ must increase in accordance with $a$ in order to maintain the observed flux. $\beta$ is less strongly correlated with $a$, but the degeneracy occurs because as $a$ increases, the peak wavelength in the SED also increases as the grain temperature decreases. Since $\beta$ affects the slope of the Rayleigh-Jeans tail of the SED, it must increase along with the position of the peak in order to obtain a steeper slope. If we were fitting for the SED alone, we would also expect $R_{i n}$ to exhibit a similarly strong degeneracy, since higher temperatures and therefore fluxes could be obtained both by bringing the grains closer to the star and by shrinking the grains. However, the inner radius is well-constrained by our spatially resolved data, so there is only a mild correlation between $R_{i n}$ and $a$, as shown in Figure 5.

It is also necessary to check that the underlying assumptions of our model do not bias these results. In Sections 4.2.1 and 4.2.2 we investigate the robustness of the best-fit results to perturbations in our assumptions about belt temperature and ring width. Finally, it is important to consider our results in the context of the scattered light morphology. The ring radius we derive is consistent with the radius reported from scattered light observations (61.25 $\pm 0.85 \mathrm{AU}$; Buenzli et al. 2010), differing by $2.6 \sigma$. This result hints at wavelength-dependent structure, in the sense that if the millimeter emission traced the scattered light precisely, the ring radius should be noticeably larger to reflect the contribution from the scattered light wings. To quantify the spatial distribution of the millimeter-wavelength emission, we perform a visibility-domain analysis of the millimeter morphology in Section 4.3, using a toy model that incorporates constraints from the scattered light emission to decompose the emission into ring and streamer components.

\subsubsection{Effect of Assumed Belt Temperature}

As discussed above, a simple single grain size fit to the data is incapable of reproducing the short-wavelength flux in the IRS spectrum. This additional flux necessitates either a population of hot grains substantially smaller than the characteristic grain size (and therefore substantially smaller than the blowout size for this system), or the addition of an inner warm dust belt. While there are very few data to constrain belt properties, we conducted a brief exploration of the effect of our assumed $100 \mathrm{~K}$ belt temperature on the derived disk parameters by running a separate MCMC chain with the tempera- 

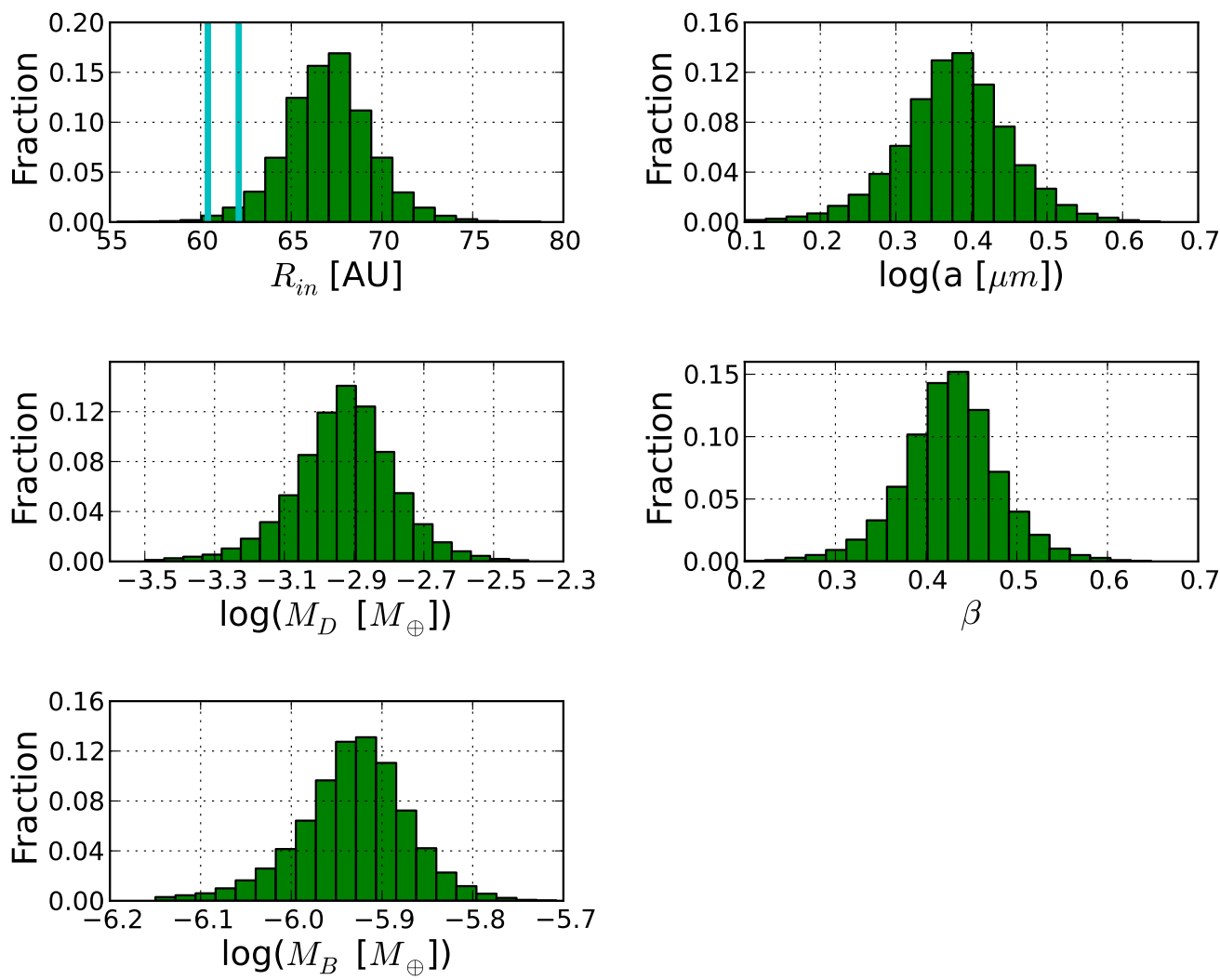

FIG. 3.- Probability distributions for the model parameters as derived from the MCMC chain in the case of a narrow ring $(w / R=5 \%)$. The height of each bar represents the fraction of the models that fall into its respective bin. In the $R_{i n}$ plot, the cyan vertical lines represent the $\pm 1 \sigma$ range in radius derived in Buenzli et al. (2010).

ture of the inner belt set to $80 \mathrm{~K}$ rather than $100 \mathrm{~K}$. The center column of Table 3 displays the results of the best fit.

The only noticeable change occurs in $M_{B}$, which increases to compensate for the decreased temperature. The visibilities fix $R_{i n}$, the SED peak fixes $a$ and $M_{D}$, and the slope of the Wien tail fixes $\beta$. This model fit deviates in quality of fit from the $100 \mathrm{~K}$ model only by $0.6 \sigma$. The available data are therefore evidently not sufficient to strongly constrain the temperature of the warm belt, but this confirms the robustness of our analysis of the outer, cold debris disk, independent of the assumed belt temperature.

\subsubsection{Effect of Disk Width}

While the data are consistent with a narrow ring centered at the radius of the scattered light ring, we also investigate a scenario in which the width is fixed to $65 \mathrm{AU}$ to determine whether the millimeter data can constrain the width of the ring. This value was chosen so that $w / R \sim 1$. The right column of Table 3 displays the results.

The best-fit broad belt has a central radius of $71 \pm 3$ AU from the star (although due to the falloff of surface density with radius, more of the emission is concentrated closer to the star), which is consistent with the radius of the narrow ring fit within 2 sigma. This model differs in quality of fit from the best-fit narrow ring model only by $0.6 \sigma$. Our spatial resolution - nominally $\sim 80 \mathrm{AU}$ with natural weighting, but including shorter baselines that provide information on smaller spatial scales - is therefore evidently not sufficient to distinguish between a wide and a narrow millimeter belt, although it is notable that in both the wide and narrow disk fit, the disk is centered on the location of the scattered light ring to within the uncertainties.

\subsection{Are there Large Grains in the Streamers?}

The swept-back "wings" are the most remarkable features of the debris disk around HD 61005. Particularly prominent in scattered light, they contribute slightly more than half the flux at short wavelengths. While most theoretical interpretations of the streamers focus on dynamics of small grains, large grains that dominate millimeter maps are far better tracers of gravitational dynamics and are therefore useful in distinguishing between mechanisms that might sculpt this striking morphology. The relative contribution of the flux at infrared and millimeter wavelengths provides insight into the grain size distribution in the wings and the streamers.

Since this is a purely geometric problem and we cannot reliably distinguish between SED contributions from the disk and the streamers, we perform the analysis on the visibilities only. We therefore depart from the analysis 

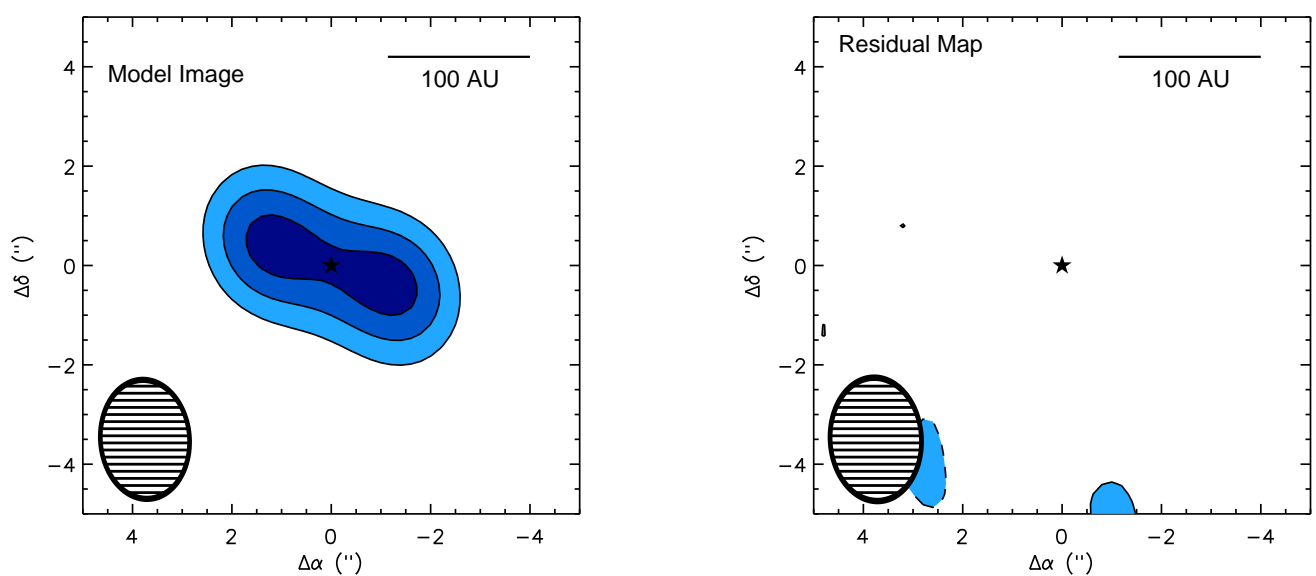

FIG. 4. - The best fit observed model image and corresponding residual map. For comparison with Figure 1$][3,5,7] \times 0.34$ mJy contours are drawn in the observed model image. In the residual map, the $+2 \sigma$ contours are drawn with solid lines, while the $-2 \sigma$ contours are drawn with dashed lines. The residual emission does not exceed the $3 \sigma$ level.

strategy of Section 4.1 and instead use a toy model that incorporates constraints on the disk structure from the observed scattered light morphology. Unlike the MCMC analysis in Section 4.2 above, we fix as many parameters as possible to match the scattered light. To maintain consistency with Buenzli et al. (2010), we fix the inner radius of the disk to $61.25 \mathrm{AU}$ and the width to the original narrow value. We set the angle that the streamers make with the plane of the disk to $23^{\circ}$, and set the flux density power law to that observed in scattered light: $F \propto r^{-4.4}$ (Buenzli et al. 2010).

We compute a grid that varies the total flux in the image and the streamer contribution. For each value of the streamer contribution between zero and one, we search for the value of the total flux between 5 and 9 mJy that minimizes the $\chi^{2}$ value between the data and model. Each of these ranges of parameters was divided into 50 steps, such that the streamer contribution was explored with a step size of $2 \%$, while the total flux was explored with a step size of $0.08 \mathrm{mJy}$. We then calculate the statistical deviation of each model from the global minimum. Figure [6 displays the results. The deviation from the global minimum $\chi^{2}$ value, expressed as the number of standard deviations corresponding to the probability with which the model is a worse fit than the global best-fit value, is plotted against the percentage of the total flux in the image that originates in the streamers (with the remainder of the flux originating from the ring). The observed streamer contribution from the scattered light data is indicated by a vertical red line. The results demonstrate that a scenario in which the millimeter emission traces the morphology of the scattered light, including the swept-back wings, is ruled out at the $4 \sigma$ level. The data are of insufficient quality to draw fine distinctions about whether some smaller fraction of the millimeter flux might originate from the streamers, but they are certainly consistent with a scenario in which the large grains responsible for the $1.3 \mathrm{~mm}$ emission are confined exclusively to the scattered light ring. The implications of this result will be discussed in more detail in Section 5.2 below.

\section{DISCUSSION}

\subsection{Dust Grain Properties}

The best-fit grain size in the fiducial model is $a=2.4 \mu \mathrm{m}$. This value is somewhat smaller than for other debris disks analyzed using similar methods (see, e.g., Williams et al. 2004; Hughes et al. 2011); the relatively small grain size reflects the high temperatures indicated by the peak wavelength of the SED, which is inconsistent with blackbody equilibrium temperature at the radii indicated by the millimeter and scattered light images. The blow-out grain size for HD 61005 due to radiation pressure is roughly $1 \mu \mathrm{m}$, estimated using the relationship $a_{\text {blowout }}=3 L_{*} / 16 \pi G M_{*} c \rho$, where $L_{*}$ is the stellar luminosity, $M_{*}$ is the mass of the star, $c$ is the speed of light, and $\rho$ is the density of a dust grain. This relationship results from balancing the radiation pressure force against the gravitational force for a particle on a circular orbit (which is a factor of two easier to remove from the potential well than a stationary dust grain), including a factor of 0.5 to estimate the effects of a realistic albedo and radiative efficiency of a silicate dust grain (Backman \& Paresce 1993).

It is clear from the ensemble of models in our fitting process that the small grain size primarily reflects the need for the grains to attain a temperature high enough to match the peak wavelength of emission while being located in a ring roughly 60-70 AU from the central star. A simple estimate of dust temperature using Wien's Law and the approximate peak wavelength of the blackbody predicts a dust temperature of about $43 \mathrm{~K}$, while the temperature predicted by Equation 4 is $50 \mathrm{~K}$ for grains with the best-fit size and emission efficiency. As a sanity check, we performed a "chi-by-eye" fit to the SED using a complete radiative transfer code with a realistic grain size distribution (MCFOST; Pinte et al.|2006, 2009). We obtained a reasonable fit with a minimum grain size of $2.5 \mu \mathrm{m}$. The ring is assumed to extend from 60 to $63 \mathrm{AU}$, with a surface density power law index $p=0.5$ and a total mass in dust of $3.3 \times 10^{-4} \mathrm{M}_{\oplus}$. The assumed dust composition is compact astrophysical silicates (Draine 2003), with a distribution spanning the $2.5 \mu \mathrm{m}-1 \mathrm{~cm}$ range and a -3.5 power law index appropriate for a collisional cascade. However, we did not pursue MCMC minimization 

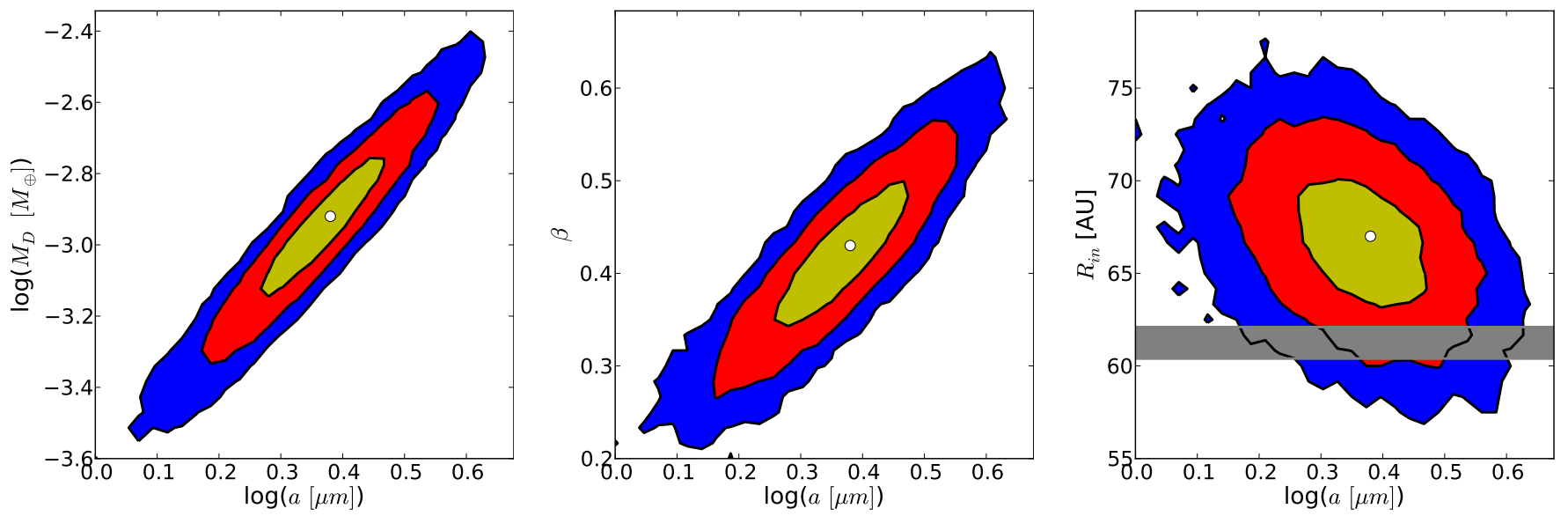

FIG. 5. - The probability distribution of an MCMC chain in the $\log (a)-\log \left(M_{D}\right), \log (a)-\beta$, and the $\log (a)-R_{\text {in }}$ planes. Contours enclose $68.2 \%(1 \sigma), 95.4 \%(2 \sigma)$, and $99.6 \%(3 \sigma)$ of the models, and the white circle marks the position of the global minimum $\chi^{2}$. Here, the degeneracy between $a, M_{D}$, and $\beta$ is readily apparent; we also note that the degeneracy with $R_{i n}$ is broken with the use of the spatially resolved visibilities. The grey rectangle in this final plot depicts the value of $R_{\text {in }}$ determined from scattered light in Buenzli et al. (2010). The best-fit radius using the millimeter data is consistent with the scattered light radius to within the uncertainties.

with this code due to the computational intensity of the task. We mention it here only to indicate that it is possible to reproduce the SED (although not the near-IR excess that we attribute to the warm belt) with a realistic grain size distribution that includes only grains larger than the blowout size.

It is also worth noting that SED modeling has been notoriously difficult due to the complexity of the system. Previous SED models using single-blackbody fits have resulted in disk radius estimates between 16 (Hines et al. 2007) and $96 \mathrm{AU}$ (Roccatagliata et al. 2009), depending on the method and assumed grain properties, while a slightly more complex extended disk model by Hillenbrand et al. (2008) predicted a disk that stretches between radii of 8.6 and $41 \mathrm{AU}$. To some extent the small radii indicate the presence of hot dust close to the star, which we model as an unresolved $100 \mathrm{~K}$ blackbody. It would be difficult to reproduce this shortwavelength excess emission using only small dust grains, since the maximum temperature of astrosilicate grains at the radius of the scattered light ring is $70 \mathrm{~K}$, and furthermore very small grains are ruled out by the lack of solid state features in the Spitzer IRS spectrum. However, the resolved observations confirm that even the outer belt is significantly hotter than its blackbody equilibrium temperature. As discussed by Booth et al. (2013), this mismatch in sizes is typical of the effort to deduce debris disk sizes from SED fitting; in the particular case of HD 61005 , increasing the porosity of the grains could help account for the high temperatures indicated by the SED. It is also interesting to note that some polarimetric observations of debris disks seem to require high grain porosity to explain the observed properties of the scattered light (e.g., Graham et al. 2007).

\subsection{Comparison of Millimeter and Scattered Light Morphology}

When considering the morphology of the millimeterwavelength emission, the most salient question is how it compares with the structure observed in scattered light. Based on the ISM-driven mechanism for creating the swept-back wings of the Moth (Maness et al. 2009; Debes et al. 2009) and the size-dependent response of dust grains to such a mechanism (Marzari \& Thébault 2011), we expect that the millimeter grains would be confined to the thin parent-body ring at $61 \mathrm{AU}$ radius and absent from the swept-back features. Indeed, the analysis in Section 4.3 demonstrates that a scenario in which the millimeter emission traces the morphology of the scattered light wings is ruled out at the $4 \sigma$ level. While this analysis is rudimentary, it is certainly suggestive that we are observing wavelength-dependent structure. The small grains responsible for scattered light appear to form the bulk of the material in the streamers, while the larger grains that dominate the $1.3 \mathrm{~mm}$ emission are confined primarily to the narrow ring observed in scattered light.

Due to the relatively low signal-to-noise of the SMA observations we are as yet unable to decisively rule out an alternative scenario, in which millimeter grains at least to some extent trace the spectacular scattered light wings. If some of the emission from large grains does in fact originate from the wings, it would provide an indication that large grains are perturbed (to a lesser extent) by the same mechanism that is responsible for the swept-back structure observed in scattered light. Given the theoretical work by Marzari \& Thébault (2011) indicating that large grains should remain unperturbed by ISM interactions, this would suggest that an alternative mechanism is responsible for the wings of the Moth. An eccentric perturber - for example, an unseen planet embedded in the disk - may also be capable of breaking the symmetry or the disk and causing the bowl-shaped appearance of the scattered light (M. Fitzgerald, in prep). Secular interactions can cause grains to acquire both a forced eccentricity (Wyatt et al. 1999) and inclination, the magnitude of which is dependent on the ratio $\beta$ of radiation pressure to gravity. Larger grains should therefore exhibit less extreme swept-back structure, although the offset of the disk center from the star position should 


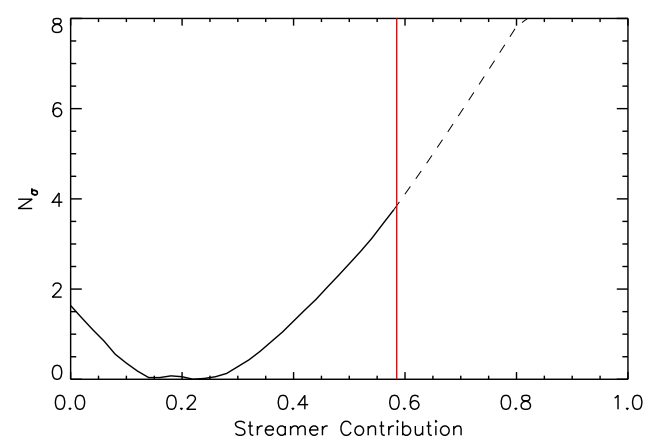

FIG. 6. - Deviation from the best fit model as a function of the fraction of millimeter-wavelength emission contributed by the streamers. The deviation is expressed in units of standard deviations (derived from $\Delta \chi^{2}$, assuming a Gaussian probability distribution). This plot effectively shows the fraction of $1.3 \mathrm{~mm}$ emission contributed by the streamers in the best-fit model (the minimum is near 0.2) and the level at which other flux fractions are ruled out in the context of our toy model. The data are consistent with a scenario in which the bulk of the millimeter emission originates from the narrow scattered light ring. The value corresponding to a scenario in which the millimeter emission traces the scattered light morphology is marked with a vertical red line, and is ruled out at the $\approx 4 \sigma$ level.

persist at millimeter wavelengths. Higher-resolution observations of the millimeter emission would therefore be advantageous in order to further disambiguate the physical processes shaping the disk.

The somewhat ambiguous morphology of the millimeter-wavelength emission should be considered in the context of hints that the HD 61005 system may host at least one planet. The system has been considered from several different planet-related perspectives. Setiawan et al. (2008) included HD 61005 in an RV search for planetary companions around nearby young star, but report that the observed variability is consistent with stellar activity rather than planets; however, the length of the survey does not appear to be sufficient to detect planets orbiting at tens of $\mathrm{AU}$ from the central star. Watson et al. (2011) include HD 61005 in a sample of debris disks with known viewing geometries, for which they estimate the inclination of the stellar rotation to the line of sight and search for evidence of misalignment. They find that debris disks, including HD 61005, are generally well aligned with the rotation axes of their host stars. The Buenzli et al. (2010) scattered light study indicates that the ring is offset from the star position by at least $2.75 \pm 0.85 \mathrm{AU}$ (in projection onto the sky plane, along the major axis only), indicative of eccentricity. They also note a pronounced brightness asymmetry between the NE and SW components of the ring that is almost certainly due to density enhancements. The upper limit on companion mass in the LOCI image is below the deuterium-burning limit, varying between roughly 3 and $6 \mathrm{M}_{\mathrm{Jup}}$ between the inner working angle and the ring radius. It is not yet clear whether the position offset and density enhancements could be caused by a planet, or whether they could be caused by the same ISM interaction that might be producing the streamers. However, the $1.6 \mu \mathrm{m}$ wavelength of the VLT observations presented in Buenzli et al. (2010) approaches the grain population discussed in Marzari \& Thébault (2011), which is too large to be effectively sculpted by the ISM. The near-IR eccentricity and asymmetric density distributions are suggestive that a mechanism other than the ISM, possibly including planets, may be needed to explain all the observed features of the system. However, it should be emphasized that the millimeter emission alone is so far fully consistent with an ISM-sculpted disk morphology.

\section{SUMMARY AND CONCLUSIONS}

We have spatially resolved the dust continuum emission from the debris disk around HD 61005 at a wavelength of $1.3 \mathrm{~mm}$. We observe a double-peaked structure consistent with an optically thin disk viewed close to edge-on. A simultaneous analysis of the spectral energy distribution and millimeter-wavelength visibilities demonstrates that the dust is hotter than the expected blackbody equilibrium temperature given the relatively large radial extent of the ring resolved in scattered light and millimeter continuum emission. This is indicative of the presence of a substantial quantity of small grains, some of which are likely close to the blow-out size.

We also investigate the morphology of the millimeterwavelength emission, particularly in comparison with the scattered light observations. Our MCMC analysis suggests that the millimeter emission arises from roughly the same stellocentric distance as the thin ring observed in scattered light. This is confirmed by a visibility-domain analysis of the millimeter emission compared with a toy model based on the observed features of the scattered light emission. In the context of our toy model, a scenario in which the millimeter morphology traces the scattered light flux distribution is ruled out at the $4 \sigma$ level. This result is suggestive of wavelength-dependent structure, in which the large grains remain in the parent body ring, while small grains are preferentially affected by the perturbation responsible for sculpting the scattered light wings. Such grain size segregation is consistent with theoretical expectations for an ISM-sculpted disk. The sensitivity and resolution of current observations is insufficient to provide a firm conclusion on the detailed morphology of the millimeter emission; hence these results remain merely suggestive. However, these investigations pave the way for more sensitive future observations, for example with the Atacama Large Millimeter/Submillimeter Array (ALMA) currently nearing the end of its construction phase, and ripe to contribute to this exciting field.

We thank Holly Maness, who obtained the early data sets used in this paper and established detection. We are also grateful to Esther Buenzli for kindly providing her LOCI image used in Figure 1. A.M.H. is supported by a fellowship from the Miller Institute for Basic Research in Science.

\section{REFERENCES}

Andrews, S. M., Wilner, D. J., Hughes, A. M., Qi, C., \& Dullemond, C. P. 2009, ApJ, 700, 1502
Backman, D. E. \& Paresce, F. 1993, in Protostars and Planets III, ed. E. H. Levy \& J. I. Lunine, 1253-1304 
Blum, J. \& Wurm, G. 2008, ARA\&A, 46, 21

Bohren, C. F. \& Huffman, D. R. 1983, Absorption and scattering of light by small particles

Booth, M., Kennedy, G., Sibthorpe, B., Matthews, B. C., Wyatt, M. C., Duchêne, G., Kavelaars, J. J., Rodriguez, D., Greaves, J. S., Koning, A., Vican, L., Rieke, G. H., Su, K. Y. L., Moro-Martín, A., \& Kalas, P. 2013, MNRAS, 428, 1263

Brownlee et al. 2006, Science, 314, 1711

Buenzli, E., Thalmann, C., Vigan, A., Boccaletti, A., Chauvin, G., Augereau, J. C., Meyer, M. R., Ménard, F., Desidera, S., Messina, S., Henning, T., Carson, J., Montagnier, G., Beuzit, J. L., Bonavita, M., Eggenberger, A., Lagrange, A. M., Mesa, D., Mouillet, D., \& Quanz, S. P. 2010, A\&A, 524, L1

Burrows, C. J., Krist, J. E., Stapelfeldt, K. R., \& WFPC2 Investigation Definition Team. 1995, in Bulletin of the American Astronomical Society, Vol. 27, American Astronomical Society Meeting Abstracts, 1329

Cutri, R. M., Skrutskie, M. F., van Dyk, S., Beichman, C. A., Carpenter, J. M., Chester, T., Cambresy, L., Evans, T., Fowler, J., Gizis, J., Howard, E., Huchra, J., Jarrett, T., Kopan, E. L., Kirkpatrick, J. D., Light, R. M., Marsh, K. A., McCallon, H., Schneider, S., Stiening, R., Sykes, M., Weinberg, M., Wheaton, W. A., Wheelock, S., \& Zacarias, N. 2003, 2MASS All Sky Catalog of point sources.

Debes, J. H., Weinberger, A. J., \& Kuchner, M. J. 2009, ApJ, 702,318

Debes, J. H., Weinberger, A. J., \& Song, I. 2008, ApJ, 684, L41

Desidera, S., Covino, E., Messina, S., D'Orazi D'Orazi, V., Alcalá, J. M., Brugaletta, E., Carson, J., Lanzafame, A. C., \& Launhardt, R. 2011, A\&A, 529, A54

Draine, B. T. 2003, ApJ, 598, 1017

Draine, B. T. \& Lee, H. M. 1984, ApJ, 285, 89

Franco, G. A. P. 1990, A\&A, 227, 499

Goodman, J. \& Weare, J. 2010, Communications in Applied Mathematics and Computer Science, 5, 65

Graham, J. R., Kalas, P. G., \& Matthews, B. C. 2007, ApJ, 654, 595

Heap, S. R., Lindler, D. J., Lanz, T. M., Cornett, R. H., Hubeny, I., Maran, S. P., \& Woodgate, B. 2000, ApJ, 539, 435

Hillenbrand, L. A., Carpenter, J. M., Kim, J. S., Meyer, M. R., Backman, D. E., Moro-Martín, A., Hollenbach, D. J., Hines, D. C., Pascucci, I., \& Bouwman, J. 2008, ApJ, 677, 630

Hines, D. C., Schneider, G., Hollenbach, D., Mamajek, E. E., Hillenbrand, L. A., Metchev, S. A., Meyer, M. R., Carpenter, J. M., Moro-Martín, A., Silverstone, M. D., Kim, J. S., Henning, T., Bouwman, J., \& Wolf, S. 2007, ApJ, 671, L165

Høg, E., Fabricius, C., Makarov, V. V., Urban, S., Corbin, T., Wycoff, G., Bastian, U., Schwekendiek, P., \& Wicenec, A. 2000, A\&A, 355, L27

Hughes, A. M., Wilner, D. J., Andrews, S. M., Williams, J. P., Su, K. Y. L., Murray-Clay, R. A., \& Qi, C. 2011, ApJ, 740, 38

Hughes, A. M., Wilner, D. J., Qi, C., \& Hogerheijde, M. R. 2008, ApJ, 678, 1119

Kalas, P. 2005, ApJ, 635, L169

Kalas, P., Graham, J. R., Chiang, E., Fitzgerald, M. P., Clampin, M., Kite, E. S., Stapelfeldt, K., Marois, C., \& Krist, J. 2008, Science, 322, 1345

Lagrange, A.-M., Bonnefoy, M., Chauvin, G., Apai, D., Ehrenreich, D., Boccaletti, A., Gratadour, D., Rouan, D., Mouillet, D., Lacour, S., \& Kasper, M. 2010, Science, 329, 57

Maness, H. L., Kalas, P., Peek, K. M. G., Chiang, E. I., Scherer, K., Fitzgerald, M. P., Graham, J. R., Hines, D. C., Schneider, G., \& Metchev, S. A. 2009, ApJ, 707, 1098
Marois, C., Macintosh, B., Barman, T., Zuckerman, B., Song, I., Patience, J., Lafrenière, D., \& Doyon, R. 2008, Science, 322, 1348

Marzari, F. \& Thébault, P. 2011, MNRAS, 416, 1890

Meyer, M. R., Carpenter, J. M., Mamajek, E. E., Hillenbrand, L. A., Hollenbach, D., Moro-Martin, A., Kim, J. S., Silverstone, M. D., Najita, J., Hines, D. C., Pascucci, I., Stauffer, J. R., Bouwman, J., \& Backman, D. E. 2008, ApJ, 673, L181

Mundy, L. G., Looney, L. W., Erickson, W., Grossman, A. Welch, W. J., Forster, J. R., Wright, M. C. H., Plambeck, R. L., Lugten, J., \& Thornton, D. D. 1996, ApJ, 464, L169

Perryman, M. A. C., Lindegren, L., Kovalevsky, J., Hoeg, E., Bastian, U., Bernacca, P. L., Crézé, M., Donati, F., Grenon, M., Grewing, M., van Leeuwen, F., van der Marel, H., Mignard, F., Murray, C. A., Le Poole, R. S., Schrijver, H., Turon, C., Arenou, F., Froeschlé, M., \& Petersen, C. S. 1997, A\&A, 323, L49

Pinte, C., Harries, T. J., Min, M., Watson, A. M., Dullemond, C. P., Woitke, P., Ménard, F., \& Durán-Rojas, M. C. 2009, A\&A, 498, 967

Pinte, C., Ménard, F., Duchêne, G., \& Bastien, P. 2006, A\&A, 459, 797

Roccatagliata, V., Henning, T., Wolf, S., Rodmann, J., Corder, S., Carpenter, J. M., Meyer, M. R., \& Dowell, D. 2009, A\&A, 497, 409

Rodigas, T. J., Hinz, P. M., Leisenring, J., Vaitheeswaran, V., Skemer, A. J., Skrutskie, M., Su, K. Y. L., Bailey, V., Schneider, G., Close, L., Mannucci, F., Esposito, S., Arcidiacono, C., Pinna, E., Argomedo, J., Agapito, G., Apai, D., Bono, G., Boutsia, K., Briguglio, R., Brusa, G., Busoni, L., Cresci, G., Currie, T., Desidera, S., Eisner, J., Falomo, R., Fini, L., Follette, K., Fontana, A., Garnavich, P., Gratton, R., Green, R., Guerra, J. C., Hill, J. M., Hoffmann, W. F., Jones, T. J., Krejny, M., Kulesa, C., Males, J., Masciadri, E., Mesa, D., McCarthy, D., Meyer, M., Miller, D., Nelson, M. J., Puglisi, A., Quiros-Pacheco, F., Riccardi, A., Sani, E., Stefanini, P., Testa, V., Wilson, J., Woodward, C. E., \& Xompero, M. 2012, ApJ, 752, 57

Setiawan, J., Weise, P., Henning, T., Hatzes, A. P., Pasquini, L. da Silva, L., Girardi, L., von der Lühe, O., Döllinger, M. P., Weiss, A., \& Biazzo, K. 2008, in Precision Spectroscopy in Astrophysics, ed. N. C. Santos, L. Pasquini, A. C. M. Correia, \& M. Romaniello, 201-204

Strubbe, L. E. \& Chiang, E. I. 2006, ApJ, 648, 652

Su, K. Y. L., Rieke, G. H., Stapelfeldt, K. R., Malhotra, R., Bryden, G., Smith, P. S., Misselt, K. A., Moro-Martin, A., \& Williams, J. P. 2009, ApJ, 705, 314

Tielens, A. G. G. M. 2005, The Physics and Chemistry of the Interstellar Medium

van Leeuwen, F. 2007, A\&A, 474, 653

Watson, C. A., Littlefair, S. P., Diamond, C., Collier Cameron, A., Fitzsimmons, A., Simpson, E., Moulds, V., \& Pollacco, D. 2011, MNRAS, 413, L71

Williams, J. P., Najita, J., Liu, M. C., Bottinelli, S., Carpenter, J. M., Hillenbrand, L. A., Meyer, M. R., \& Soderblom, D. R. 2004, ApJ, 604, 414

Wilner, D. J., Andrews, S. M., \& Hughes, A. M. 2011, ApJ, 727, L42

Wilner, D. J., Andrews, S. M., MacGregor, M. A., \& Hughes, A. M. 2012, ApJ, 749, L27

Wyatt, M. C. 2006, ApJ, 639, 1153

Wyatt, M. C., Dermott, S. F., Telesco, C. M., Fisher, R. S., Grogan, K., Holmes, E. K., \& Piña, R. K. 1999, ApJ, 527, 918 